\title{
Clinical Efficacy of Single-visit Pulpectomy over Multiple-visit Pulpectomy in Primary Teeth: A Systematic Review
}

\author{
Sunny P Tirupathi ${ }^{1}$, Nirmala Krishna ${ }^{2}$, Srinitya Rajasekhar ${ }^{3}$, Sivakumar Nuvvula ${ }^{4}$
}

\begin{abstract}
Objective: The purpose of this systematic review was to compare the efficacy of single-visit pulpectomy (SVP) vs multiple-visit pulpectomy (MVP) in infected primary teeth.

Materials and methods: An extensive literature search in the English language was conducted in PICO format using MeSH terms using databases (PubMed, EBSCO, Ovid, and Cochrane) and pre-specified inclusion and exclusion criteria were applied to identify relevant studies comparing pulpectomy in single and multiple visits.

Results: Only 4 studies (3-in vivo clinical study; 1 -in vivo microbial study) sustained the final analysis and were included for critical appraisal. Results of the systematic search revealed that there are only a few studies comparing the efficacy of single-visit pulpectomy vs multiple-visit pulpectomy in infected primary teeth.

Conclusion: On the basis of the available studies, evidence favors the SVP protocol over the MVP protocol. Whenever possible the single-visit protocol can be preferred over the multiple-visit protocol. The quality of evidence available is low.

Keywords: Efficacy, Primary teeth, Pulpectomy, Visits.

International Journal of Clinical Pediatric Dentistry (2019): 10.5005/jp-journals-10005-1654
\end{abstract}

\section{InTRODUCTION}

Pulpectomy is a regular clinical procedure in pediatric dentistry, which involves removal of infected dental pulp and suitable medicament could be used to fill the canals. The key indications for pulpectomy are irreversible pulpitis and necrosis of the dental pulp. Mechanical removal of complete necrotic tissue debris from root canal of a primary tooth followed by copious cleaning and irrigation with disinfecting agents, and the chemo-mechanically prepared root canal is filled with suitable obturating material with coronal seal is called pulpectomy. ${ }^{1}$ Nonetheless, successful pulpectomy in primary teeth is characterized by the absence of signs and symptoms clinically and radiographically. Moreover, tooth status, its pathological condition and clinician expertise are the key factors for the success of pulpectomy in primary teeth.

Figini et al., reported that, there is no significant difference between single-visit and a multiple-visit root canal treatment in the terms of clinical and radiographic success in permanent teeth. ${ }^{2}$ A most recent meta-analysis on nonsurgical single-visit vs a multiple-visit endodontic treatment in permanent teeth showed that, postoperative complications of both the procedures similar. Furthermore, neither single-visit nor a multiple-visit endodontic treatment had shown superior results in terms of healing or success rate in permanent teeth. ${ }^{3}$ In contrast some authors ${ }^{4,5}$ opined that single-visit endodontic therapy is better than a multiple-visit in terms of clinical and radiographic success. Most of the pediatric dentists prefer to perform a multiple visit pulpectomy to ensure thorough serialization of root canals prior to obturation. ${ }^{6}$ Multiple visit pulpectomy (MVP) involves extirpation of the pulp tissue and placement of intra-canal medicament in the first visit followed by obturation in the subsequent visit, if the underlying pathology still persists it may require additional visits. Singlevisit pulpectomy (SVP) involves extirpation of pulp and filing the canals short of the apex to a resistance point, after irrigation and
${ }^{1}$ Department of Pedodontics and Preventive Dentistry, Malla Reddy Institue of Dental Sciences, Hyderabad, Telangana, India

${ }^{2-4}$ Department of Pedodontics and Preventive Dentistry, Narayana Dental College and Hospital, Nellore, Andhra Pradesh, India

Corresponding Author: Sunny P Tirupathi, Department of Pedodontics and Preventive Dentistry, Malla Reddy Institue of Dental Sciences, Hyderabad, Telangana, India, Phone: +91 9490549454, e-mail: dr.priyatham@gmail.com

How to cite this article: Tirupathi SP, Krishna N, Rajasekhar S, et al. Clinical Efficacy of Single-visit Pulpectomy over Multiple-visit Pulpectomy in Primary Teeth: A Systematic Review. Int J Clin Pediatr Dent 2019;12(5):453-459.

Source of support: Nil

Conflict of interest: None

final drying obturating material will be placed in same visit. ${ }^{4-6}$ However, there is no evidence to support that weather singlevisit or a multiple-visit pulpectomy treatment in primary teeth. Furthermore there is need to evaluate the studies published on single and multiple visit pulpectomy in primary teeth. Hence, the aim of the present study was to clarify whether performing pulpectomy in the primary tooth in a single visit or in multiple visits, makes any difference in term of efficacy or complications or both, based on the available literature.

\section{Null-hypothesis}

Single-visit pulpectomy is better than a multiple-visit pulpectomy.

\section{Materials and Methods}

A comprehensive and systematic search was carried out using MeSH terms in a structured PICO format (Table 1) in four databases 
Table 1: Terms in search strategy used in PICO format

\begin{tabular}{llll}
\hline Population & Intervention & Comparison & Outcome \\
\hline Primary teeth & Single-visit pulpectomy & Multiple-visit pulpectomy & Success \\
Necrotic primary teeth & Single-visit root canal therapy & Multiple-visit root canal therapy & Treatment outcome \\
Necrosed primary teeth & One-visit root canal therapy & Root canal therapy & Success rate \\
Necrotic deciduous teeth & One-visit root canal treatment & Endodontic treatment & Efficacy \\
Deciduous teeth & Single-visit root canal treatment & Multiple-visit root canal treatment & Pain \\
Dental pulp necrosis & Single-visit pediatric endodontics & Multiple-visit pediatric endodontics & Postoperative pain \\
Periapical abscess & Single-visit pulp therapy & Multiple-visit paediatric endodontics & Swelling \\
Pulpitis & Single-visit pulp treatment & Conventional pulpectomy & Inflammation \\
Irreversible pulpitis & One-visit pulpectomy & Multi-visit pulpectomy & Edema \\
Dentoalveolar abscess & Single-appointment pulpectomy & Multiple-sitting pulpectomy & Healing \\
Primary molars & Single-sitting pulpectomy & Two-visit pulpectomy & Duration of healing \\
Children & Single-session pulpectomy & Three-visit pulpectomy & \\
Nonvital & Single-time pulpectomy & Four-visit pulpectomy & \\
Pulp necrosis & One-session pulpectomy & Multiple-visit protocol & \\
Primary root canals & Single-visit protocol & Pulpectom & \\
& One-stage pulpectomy & & \\
\hline
\end{tabular}

(PubMed, Cochrane library, Ovid SP, and EBSCOhost). Articles published in the English language between January 1981 and January 2019 were considered as qualified for the search strategy. Relevant cross-references were evaluated. Grey literature, conference papers, and unpublished work are excluded. Only prospective studies were included. A hand search was performed to include any relevant articles. The last search was performed on January 2019.

\section{Search Terms}

Search terms used were as follows: "Primary teeth," "Deciduous teeth," "Dental pulp necrosis," "Periapical abscess," "Pulpitis," "Irreversible pulpitis," "Dentoalveolar abscess," "Single-visit root canal treatment," "Single-visit pediatric endodontics," "Single-visit pulp therapy," "Multiple visit root canal therapy," "Multiple visit pediatric endodontics," "Pulpectomy", "Conventional pulpectomy," "Pain, Postoperative pain," "Swelling, Inflammation," "Edema," and "Healing" were used in various combinations.

\section{Inclusion Criteria}

Human in vitro, in vivo, and ex vivo studies published from January 1981 to January 2019 that compared both single and multiple-visit pulpectomy in primary teeth were included. Randomized control trials, quasi-randomized control trials, case reports, and systematic reviews were included. Studies published in English language were included for assessment.

\section{Exclusion Criteria}

Animal studies, studies on permanent teeth, narrative reviews, conference papers, letters to the editor, retrospective studies, and studies considering either single visit or multiple visit alone in primary dentition are excluded, studies published in languages other than English.

The titles and abstracts of all reports identified through the electronic searches were evaluated independently by authors. Studies that appear to meet inclusion criteria and studies with insufficient or partial information at the title and abstract stage were included for a full-text review in four databases separately (PubMed, Cochrane, Ovid SP, and EBSCOhost). Duplicates were excluded.
Final articles that meet the requirement were included for quality appraisal; outcome assessment and critical appraisal was carried out for these selected articles. Variables that are used for outcome measurement/assessment such as clinical success and failure, radiographic success and failure, and post-operative pain (if any) were taken for evaluation. The systematic review was reported adhering to the Preferred Reporting Items for Systematic Review and Meta-Analysis Protocols (PRISMAP) statement ${ }^{7}$ (Flowchart 1).

\section{Results}

PICO search revealed a total of 137 titles from four data bases along with 2 from hand search. At preliminary level 13 duplicates were excluded. A total of 113 articles were excluded at the title and abstract stage. Most common reasons for exclusion were studies on permanent teeth and studies that follow only the SVP or MVP protocol. Totally, 13 articles were included for a full-text review (without exclusion of duplicates). Among these, 9 articles were excluded after going through full texts, and only 4 articles ${ }^{8-11}$ were available for final analysis (Table 2). All these four articles performed both single-visit and multiple-visit pulpectomy protocol in the same study. Studies with only SVP in primary dentition ${ }^{12-18}$ and studies with only MVP in primary dentition ${ }^{19,20}$ were excluded from this systematic search. Among the finally available studies, three from India, $8,10,11$ were in vivo studies and one from Brazil was in vivo study with microbiological evaluation. ${ }^{9}$

In the in vivo study from India, ${ }^{8} 40$ carious involved primary molars with no sign of abnormal mobility, swelling, or sinus tract formation, and requiring pulpectomy were randomly divided into two groups (group I: SVP, group II: MVP). In the SVP group after access opening, biomechanical preparation was carried out and irrigation was done with $2.5 \%$ sodium hypochlorite. Access cavities were sealed with a silver amalgam/glass ionomer cement after obturation in the same visit. In the MVP group, access was gained and after biomechanical preparation, root canals were dried and filled with calcium hydroxide powder mixed with normal saline and access cavities were sealed with temporary cement. After 7 days, calcium hydroxide dressing was removed with reamers and normal saline as irrigant. The root canals were dried using absorbent 
Flowchart 1: PRISMA diagram of study design

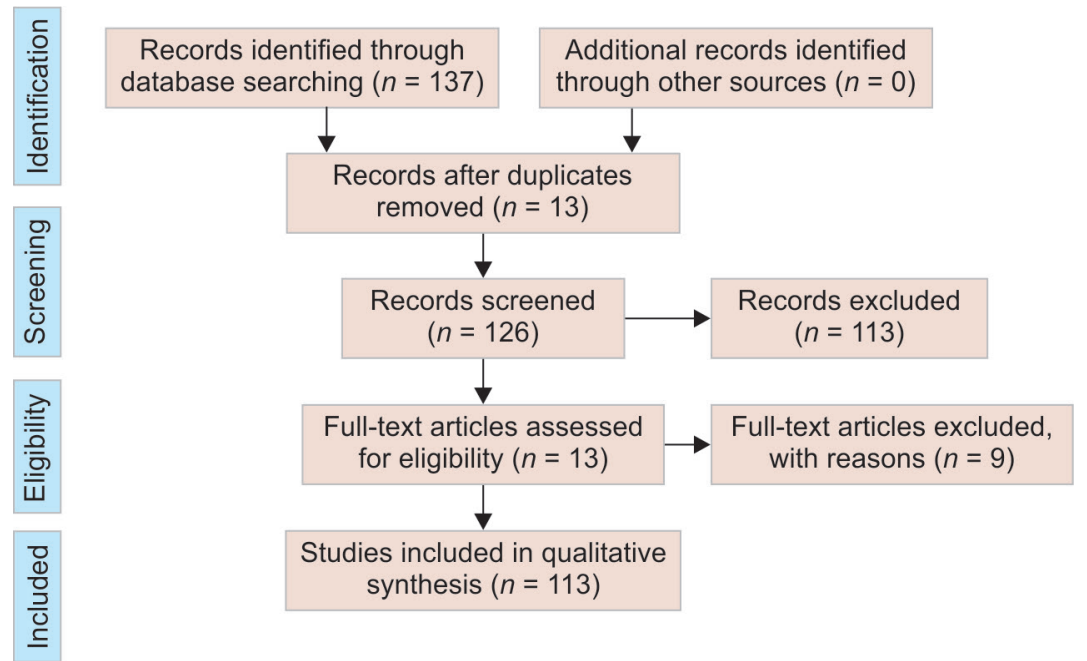

Table 2: Summary of studies available for final analysis

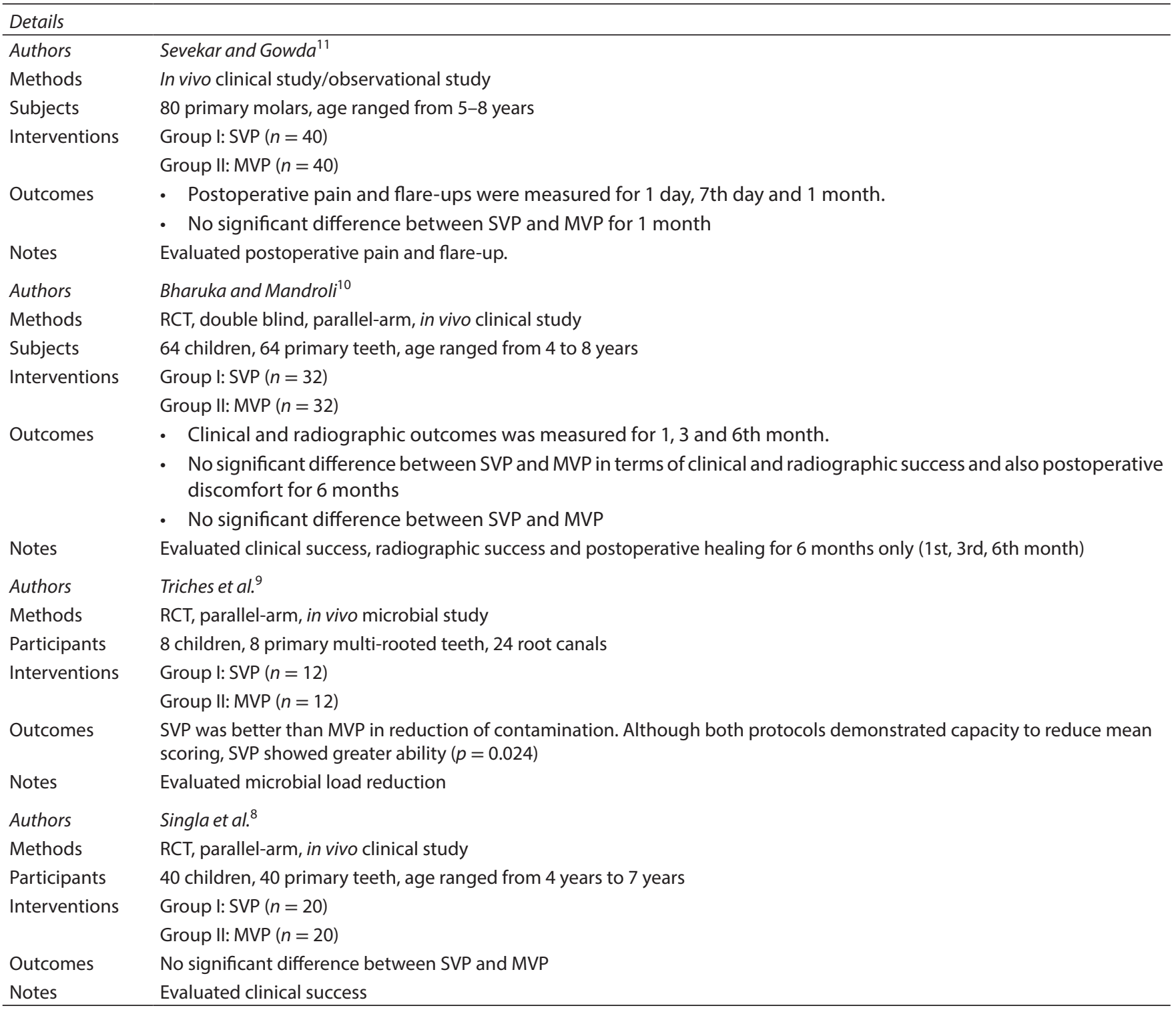

SVP, single visit pulpotomy; MVP, multi-visit pulpotomy; RCT, randmized clinical trial; bias was checked with Cochrane risk of bias tool 
paper points and obturated with zinc oxide eugenol cement using engine-driven lentulo-spirals. Access cavities were sealed with silver amalgam/glass ionomer cement. Clinical and radiographic evaluation was carried out after 1-week, 3-weeks' and 6 months' interval using the Gutmann criteria. ${ }^{12}$ At 1 week, in a follow-up of two patients (2 out of 20) reported with pain in the SVP group and MVP group, all children were asymptomatic. One patient in the SVP group reported development of intraoral sinus tract after one month; therefore this patient was shifted from SVP to MVP group and recorded as failure. At three months follow-up, all patients in both groups were asymptomatic (19 of 19 in the SVP group and 20 of 20 in the MVP group). After six months, one patient from each group was not reported and hence were excluded from the analysis. So at six months follow-up, all were asymptomatic in both groups. There was no significant difference in the failure rates between SVP and MVP group after six months' study period ( $p$ value- 0.99 ).

In an in vivo study by Triches et al., ${ }^{9} 24$ root canals of eight multiradicular primary teeth (with 3 canals each one) with pulp necrosis and periradicular lesion that are indicated for pulpectomy were randomly divided into two groups $(n=12)$ : the SVP group and the MVP group. Single-visit protocol (SVP): root canals were emptied and cleaned using only two large-caliber files, following the first one, and irrigation with $0.5 \% \mathrm{NaOCl}$ buffered with sodium bicarbonate and endo-c-prep (10\% urea peroxide, $15 \%$ Tween 80 and $75 \%$ Carbowax). Final irrigation was performed with Tergensol. Root canal filling was performed with an iodoform paste. Multiple-visit protocol (MVP): this protocol was performed in three visits, starting with a previous medication with diluted formocresol (1:5) for 7 days, to neutralize the septic content of the root canal. Emptying and shaping of the canals were performed in the second appointment, using endodontic files up to a size of 35 or 40 and irrigation with $1 \%$ sodium hypochlorite. Third appointment consisted of root canal filling with zinc oxide and eugenol cement. After access opening and before starting SVP or MVP, microbial samples were collected from all the root canals using three \#15 size sterile paper points and these were transferred to eppendorf tubes containing $0.15 \mathrm{~mL}$ of buffer solution ( $\mathrm{pH}$ 7.6). One-hundred milliliters of $0.5 \mathrm{M} \mathrm{NaOH}$ was added to each tube and the samples were dispersed using a vortex mixer. Evaluation was performed using checkerboard DNA-DNA hybridization for the identification of 40 different bacteria. This first sampling was aimed to determine the initial microbial profile. After SVP and MVP protocol, a similar bacterial analysis is carried out using checkerboard DNA-DNA hybridization. Comparison of microbial reduction between protocols revealed that there was a greater microbial reduction of mean scoring in the SVP group when compared to the MVP group, with a statistically significant difference between groups $(p=0.024){ }^{9}$

Bharuka and Mandroli ${ }^{10}$ performed a prospective double-blind parallel randomized clinical trial. The authors compared the clinical and radiographic outcome between SVP and MVP in primary teeth with apical periodontitis in 64 children (32 SVP and 32 MVP). This study reported that there was no significant differences among the study groups at a 6-month follow-up period clinically and radiographically. They have concluded that SVP is better than MVP in carious primary teeth with apical periodontitis.

Sevekar and Gowda ${ }^{11}$ conducted an in vivo prospective randomized clinical trial and authors compared a postoperative flare-up and pain incidence between SVP and MVP in primary molars. An estimated 80 children with 80 primary molars were distributed equally to study groups. Four cases in both the groups were reported post-operative pain (10\%) at one day recall. One flare-up (2.5\%) was noted in each group, $p=0.67$. None of the patients reported pain at the one week and one month follow-up. Postoperative pain was documented in five non-vital teeth (13.5\%) and three vital teeth (6.9\%). However, it was statistically not significant $(p>0.05)$. There were no differences between SVP and MVP protocols with respect to the incidence of postoperative pain. Moreover, no significant correlation found between the incidence of postoperative pain and pulp vitality.

So in these earlier-mentioned studies, two studies evaluated clinical and radiographic success between two protocols (SVP and MVP) and other study evaluated the microbial content reduction between two groups. Other studies evaluated post-operative pain and flare-ups between SVP and MVP (Table 3).

\section{RISK OF BIAS}

The Cochrane Collaboration's tool was used for assessing the risk of bias. ${ }^{7}$ Risk of bias is unclear in the study by Singla et al., as there is insufficient information about allocation concealment and blinding of personnel and blinding of clinical and radiological outcome assessment. However, outcome data was clear. Risk of bias is unclear in the study by Triches et al., ${ }^{9}$ as there is insufficient information about allocation concealment. As it is an in vivo bacterial study, blinding of personnel and blinding of clinical and radiological outcome assessment is not applicable in this situation. In a study by Sevekar, the risk of bias is unclear. ${ }^{11}$ The risk of bias is low in the study by Bharuka and Mandroli as allocation concealment was done. ${ }^{10}$ On the basis of the available studies for this review, bias is unclear to high. The quality of the existing studies is low to moderate follow-up only for 6 months; ${ }^{8}$ another study is an in vivo

Table 3: Risk of bias of finally available studies

\begin{tabular}{|c|c|c|c|c|c|c|c|}
\hline & $\begin{array}{l}\text { Random } \\
\text { sequence } \\
\text { generation } \\
\text { (selection bias) }\end{array}$ & $\begin{array}{l}\text { Allocation } \\
\text { concealment } \\
\text { (selection bias) }\end{array}$ & $\begin{array}{l}\text { Blinding of } \\
\text { participants } \\
\text { and personnel } \\
\text { (performance } \\
\text { bias) }\end{array}$ & $\begin{array}{l}\text { Blinding of } \\
\text { outcome } \\
\text { assessment } \\
\text { (detection bias) }\end{array}$ & $\begin{array}{l}\text { Incomplete } \\
\text { outcome data } \\
\text { (attrition bias) }\end{array}$ & $\begin{array}{l}\text { Selective reporting } \\
\text { (reporting bias) }\end{array}$ & Other bias \\
\hline Singla et al. ${ }^{8}$ & $?$ & + & - & + & - & + & $?$ \\
\hline Triches et al. ${ }^{9}$ & $?$ & + & + & $?$ & $?$ & + & + \\
\hline $\begin{array}{l}\text { Bharuka and } \\
\text { Mandroli }^{10}\end{array}$ & $?$ & + & - & + & + & + & $?$ \\
\hline $\begin{array}{l}\text { Sevekar and } \\
\text { Gowda }{ }^{11}\end{array}$ & + & + & + & + & + & $?$ & + \\
\hline
\end{tabular}

+, Low bias; -, High bias; ?, Unclear bias 
Table 4: Objectives and conclusion of studies finally evaluated

\begin{tabular}{|c|c|c|c|}
\hline Author & Study & Objective & Conclusion \\
\hline Singla et al..$^{8}$ & In vivo & $\begin{array}{l}\text { To determine clinical success rate of single visit } \\
\text { verses multiple visit root canal treatment in cariously } \\
\text { exposed vital primary molars. }\end{array}$ & $\begin{array}{l}\text { Multiple visit and single visit root canal treatment } \\
\text { demonstrated almost equal success }\end{array}$ \\
\hline Triches et al. $^{9}$ & In vitro & $\begin{array}{l}\text { To determine the efficacy of chemical-mechanical } \\
\text { procedures of two endodontic protocols for septic } \\
\text { content reduction of root canals from primary teeth } \\
\text { with pulp necrosis and periradicular lesion }\end{array}$ & $\begin{array}{l}\text { Single visit protocol showed greater efficacy in } \\
\text { reducing endodontic infection }\end{array}$ \\
\hline $\begin{array}{l}\text { Bharuka and } \\
\text { Mandroli }^{10}\end{array}$ & In vivo & $\begin{array}{l}\text { To compare and evaluate the clinical and radiographic } \\
\text { outcome of single- vs two-visit pulpectomy treatment } \\
\text { in primary teeth with apical periodontitis at the end of } \\
6 \text {-month healing period. }\end{array}$ & $\begin{array}{l}\text { Single-visit pulpectomy can be considered as a viable } \\
\text { option for the treatment of primary teeth with apical } \\
\text { periodontitis }\end{array}$ \\
\hline \multirow[t]{2}{*}{$\begin{array}{l}\text { Sevekar and } \\
\text { Gowda }\end{array}$} & In vivo & $\begin{array}{l}\text { To compare the incidence and intensity of } \\
\text { postoperative pain and flare-ups between single- and } \\
\text { multiple visit pulpectomy in primary molars. }\end{array}$ & $\begin{array}{l}\text { Majority of patients in both groups reported no pain } \\
\text { or only minimal pain within } 24 \text { hours of treatment. }\end{array}$ \\
\hline & & $\begin{array}{l}\text { To correlate the preoperative status of the pulp to } \\
\text { postoperative pain and flare-ups }\end{array}$ & $\begin{array}{l}\text { No differences between single- and multi visit } \\
\text { treatment protocols with respect to the incidence of } \\
\text { postoperative pain. }\end{array}$ \\
\hline
\end{tabular}

microbial study that tested only microbial content reduction and it did not evaluate clinical or radiographic success, and the results may or may not have validity in clinical situations. The details of the risk of bias for finally available studies were shown in Table 4.

\section{Discussion}

The primary objective of performing pulpectomy in an infected primary teeth is to eliminate microorganisms and prevent reinfection, thereby creating a favorable environment for healing of periradicular tissues and reducing the pain and discomfort to the child. Elimination of microorganisms from root canal is achieved by cleaning and shaping, which could be carried out with two protocols (SVP and MVP). Prevention of reinfection is achieved by proper threedimensional obturation of primary root canals by either techniques. Many studies proved that even after proper and thorough cleaning and shaping of root canal, it is impossible to achieve bacteria-free primary root canal system. ${ }^{13}$ The main hypothesis behind MVP is that inter appointment dressing has the ability to reduce microbial load in primary teeth ${ }^{12}$ and in permanent teeth, ${ }^{14-28}$ but some studies in permanent dentition have shown that calcium hydroxide fails to produce sterile root canals and even allows regrowth in some cases. ${ }^{18,19}$ Similar results in primary dentition were obtained. ${ }^{20}$ Following root canal dressing, the prevalence of microorganisms inside the root canal did not change; however, there was a decrease in the number of bacteria. ${ }^{20}$ Even if inter-appointment dressing could reduce the microbial load, i.e. (negative microbial culture) it does not guarantee healing in all cases. There is no significant association between negative microbial culture and healing rates success in root canal of both primary and permanent teeth. ${ }^{21-23} \mathrm{Few}$ studies in permanent dentition reported MVP without intracanal medicament in the inter-appointment period ${ }^{22,24-26}$ and MVP with intracanal medicament was reported by few authors. ${ }^{22,27-29} \mathrm{MVP}$ in primary dentition was favored by few authors. ${ }^{30-39}$

Advantages of SVP in primary teeth are that its procedural steps are simple and it aims at cleaning of root canals. In contrast, MVP protocol in primary teeth needs 3-4 visits to perform, each visit involves anesthesia, absolute isolation, and temporary crown sealing, which can be lost between visits and MVP consumes more time. Less visits and minimal radiation exposures are added benefits of SVP and SVP in primary teeth was favored by few authors. ${ }^{6-11,40-44}$
Both SVP and MVP are carried out in one study. ${ }^{6}$ In the final included studies, ${ }^{8-11}$ selection of subjects were based on their age (mean age of 6 ) and status of dentition. In both studies, multirooted primary teeth were selected. Singla et al., ${ }^{8}$ selected patients that presented with dental carious involving pulp, showing no sign of abnormal mobility, swelling or sinus tract formation, and requiring pulpectomy. Duration between the first and subsequent appointments were not mentioned for MVP in other two studies. ${ }^{10,11}$ The number of canals were taken into consideration in Triches study but the number of tooth were considered in all the other studies. Sample size calculation was mentioned in two studies., ${ }^{9,11}$ However, sample size calculation was not mentioned in the other two studies. ${ }^{8,10}$

Singla et al. ${ }^{8}$ observed no significant difference between clinical and radiographic success rates between SVP and MVP protocol $(p>0.05)$. Brazilian study ${ }^{9}$ indicate that there is a significant reduction in the bacterial load in the teeth that treated with SVP protocol when compared with MVP group and the results were statistically significant $(p<0.05)$. Whilst, Bharuka and Mandroli ${ }^{10}$ there was no statistically significant difference in clinical and radiographic outcomes between the SVP and MVP group ( $p$ value0.66). Similarly Sewekar and Gowda ${ }^{11}$ also indicate that there is no significant difference in the post-operative pain and flare ups between SVP and MVP group ( $p>0.05)$.

In the SVP group, after access opening irrigation was performed using sodium-hypochloride-based irrigants in both the studies. One study used $1 \%$ sodium hypochlorite as irrigant..$^{10}$ Two studies used $2.5 \%$ sodium hypochlorite ${ }^{8,11}$ as irrigant, whereas the other used $0.5 \% \mathrm{NaOCl}$ buffered with sodium bicarbonate and endo-cprep. ${ }^{8}$ Sevekar and Gowda used $3 \%$ sodium hypochlorite followed by saline as irrigant. ${ }^{10}$

In the MVP group, interappointment duration was similar for two studies ( 7 days) $)^{8,9}$ and other two studies did not specify an interappointment duration. ${ }^{10,11}$ However, MVP protocol differed in all the studies. In the study by Sevekar and Gowda, ${ }^{11}$ two-visit protocol was used, with formocresol as inter-appointment medicament. Two-visit protocol was also followed in two studies using calcium hydroxide as interappointment dressing. ${ }^{8,10}$ But three-visit protocol was adopted in other studies and they did not mention the exact inter appointment dressing. ${ }^{9}$ In three studies, inter-appointment 
dressing was given in the first visit after bio-mechanical preparation of root canal was accomplished; $8,10,11$ however, other study used interappointment dressing initially in the first visit followed by bio-mechanical preparation in the second visit. ${ }^{9}$

Post-operative pain and flare-ups were evaluated in the study by Sevekar and Gowda. ${ }^{11}$ Clinical and radiographic success rates were evaluated in two studies. ${ }^{8,10}$ Success and failure rates of this study can be due to a variety of factors such as the protocol used, the type of irrigant used, and also the obturating material used. Other studies were aimed at evaluating microbial load reduction between two protocols (SVP and MVP). The efficacy of protocols in this study is not dependent on the obturation material used. The role of obturation materials in the both studies cannot be compared because one study aimed at evaluating clinical and radiographic success rates and other study evaluated the microbial load reduction after these two protocols. When compared to the MVP protocol, where there was no clinical and radiographic failure rates (0/19), the SVP protocol in Singla et al., study revealed only one failure out of 19 subjects at 6 months (1/19) owing to the development of intra oral sinus tract, which was later treated by MVP protocol. So the difference in the failure rates were not statistically significant. In a study, SVP protocol outperformed MVP protocol in bacterial load reduction. ${ }^{9}$

In an Indian study, clinical and radiographic success were evaluated after 1 week, 1 month, 3 months, and six months. ${ }^{9}$ In another study, follow-up evaluation is not applicable. ${ }^{9}$ One was dropped out in the SVP and one was dropped out in the MVP group in Singla's study. In Triches' study, primary root canals with pulp necrosis and periradicular lesion were divided into two treatment groups (SVP and MVP); they reported that SVP resulted in better reduction of bacterial load when compared to MVP, which was statistically significant. Sevekar and Gowda also favored the SVP protocol as there is no difference in postoperative flare-ups and pain in both SVP and MVP groups. Singla and Bharuka, on the basis of their in vivo study findings, reported that there is no significant difference between SVP and MVP in terms of clinical and radiographic success for six months in primary dentition. . $^{8,10}$ Studies in permanent teeth reveal that irrespective of the condition of tooth, there is no significant difference in the healing and success rates between single-visit root canal treatment and multiple-visit root canal treatment. ${ }^{23,45,46}$ There is no significant difference in the post-operative complications between two groups; ${ }^{47}$ therefore, single-visit root canal treatment is efficacious and can be preferred over multiple-visit root canal treatment in permanent teeth. On the basis of the available studies for primary teeth, we can favor the SVP protocol over the MVP protocol in primary carious molars requiring pulpectomy.

\section{ConCLUSION}

However, more evidence is required to choose SVP or MVP protocol to perform pulpectomy in primary teeth. SVP may be recommend for the pulpectomy in primary teeth over MVP protocol may due to MVP takes more visits with addition pain management and radiation exposures. A well-planned randomized control trial with an adequate sample size comparing single-visit pulpectomy and multiple-visit pulpectomy in primary teeth with a proper follow up for adequate time (12-18 months) is required. Evaluating short-term and long-term complications of both protocols would contribute to confidently apply their results in clinical practice.
Why this paper is important to pediatric dentists:

- Single-visit pulpectomy is cost effective for both patients and pediatric dentists.

- No need of repeated local anesthesia administration in the SVP protocol.

- Reduced mechanical (rubber dam isolation, repeated filing) and chemical trauma (chemical irrigants) to the primary dentition in the SVP protocol.

\section{References}

1. Smail-Faugeron V, Courson F, et al. Pulp treatment for extensive decay in primary teeth. Cochrane Database Syst Rev 2018 May;5:CD003220. DOI: $10.1002 / 14651858$.

2. Figini $L$, Lodi $G$, et al. Single vs multiple visits for endodontic treatment of permanent teeth: a Cochrane systematic review. J Endod 2008 Sep;34(9):1041-1047. DOI: 10.1016/j.joen.2008.06.009.

3. Wong AW, Zhang C, et al. A systematic review of nonsurgical singlevisit vs multiple-visit endodontic treatment. Clin Cosmet Investig Dent 2014;6:45-56. DOI: 10.2147/CCIDE.S61487.

4. Hargreaves KM. Single-visit more effective than multiple-visit root canal treatment. Evid Based Dent 2006;7(1):13-14. DOI: 10.1038/ sj.ebd.6400372.

5. Sathorn C, Parashos P, et al. Effectiveness of single- vs multiple-visit endodontic treatment of teeth with apical periodontitis: a systematic review and meta-analysis. Int Endod J 2005 Jun;38(6):347-355. DOI: 10.1111/j.1365-2591.2005.00955.x.

6. Ozalp N, Saroglu I, et al. Evaluation of various root canal filling materials in primary molar pulpectomies: an in vivo study. Am J Dent 2005 Dec;18(6):347-350.

7. Moher D, Shamseer L, et al. Preferred reporting items for systematic review and meta-analysis protocols (PRISMA-P) 2015 statement. Syst Rev 2015;4(1):1-9. DOI:10.1186/2046-4053-4-1.

8. Singla R, Marwah N, et al. Single visit vs multiple visit root canal therapy. Int J Clin Pediatr Dent 2008;1(1):17-24. DOI: 10.5005/ jp-journals-10005-1004.

9. Triches TC, de Figueiredo LC, et al. Microbial reduction by two chemical-mechanical protocols in primary teeth with pulp necrosis and periradicular lesion - an in vivo study. Braz Dent J 2014;25(4): 307-313. DOI: 10.1590/0103-6440201302416.

10. Bharuka SB, Mandroli PS. Single- vs two-visit pulpectomy treatment in primary teeth with apical periodontitis: A double-blind, parallel group, randomized controlled trial. J Indian Soc Pedod Prev Dent 2016;34(4):383-390. DOI: 10.4103/0970-4388.191429.

11. Sevekar SA, Gowda SHN. Postoperative pain and flare-ups: comparison of incidence between single and multiple visit pulpectomy in primary molars. J Clin Diagn Res 2017;11(3):ZC09-ZC12. DOI: $10.7860 / J C D R / 2017 / 22662.9377$.

12. Gutmann JL. Clinical, radiographic, and histologic perspectives on success and failure in endodontics. Dent Clin North Am 1992;36(2):379-392.

13. Faria G, Nelson-Filho $P$, et al. Antibacterial effect of root canal preparation and calcium hydroxide paste (Calen) intracanal dressing in primary teeth with apical periodontitis. J Appl Oral Sci 2005;13(4):351-355. DOI: 10.1590/S1678-77572005000400007.

14. de Souza CAS, Teles RP, et al. Endodontic therapy associated with calcium hydroxide as an intracanal dressing: microbiologic evaluation by the checkerboard DNA-DNA hybridization technique. J Endod 2005;31(2):79-83. DOI: 10.1097/01.DON.0000133157.60731.3F.

15. Byström A, Sunvqvist $G$. The antibacterial action of sodium hypochlorite and EDTA in 60 cases of endodontic therapy. Int Endod J 1985;18(1):35-40. DOI: 10.1111/j.1365-2591.1985.tb00416.x.

16. Rodrigues HH, Biffi JCG. A histobacteriological assessment of nonvital teeth after ultrasonic root canal instrumentation. Endod Dent Traumatol 1989;5(4):182-187. DOI: 10.1111/j.1600-9657.1989.tb00357.x. 
17. Sjögren U, Sundqvist G. Bacteriologic evaluation of ultrasonic root canal instrumentation. Oral Surg Oral Med Oral Pathol 1987;63(3):366-370. DOI: 10.1016/0030-4220(87)90208-8.

18. Kvist T, Molander A, et al. Microbiological evaluation of one-and two-visit endodontic treatment of teeth with apical periodontitis: a randomized, clinical trial. J Endod 2004;30(8):572-576. DOI: 10.1097/01.DON.0000121607.87969.6E.

19. Orstavik D, Kerekes $K$, et al. Effects of extensive apical reaming and calcium hydroxide dressing on bacterial infection during treatment of apical periodontitis: a pilot study. Int Endod J 1991;24(1):1-7. DOI: 10.1111/j.1365-2591.1991.tb00863.x.

20. Ito IY, Junior FM, et al. Microbial culture and checkerboard DNADNA hybridization assessment of bacteria in root canals of primary teeth pre- and post-endodontic therapy with a calcium hydroxide/ chlorhexidine paste. Int J Paediatr Dent 2011;21(5):353-360. DOI: 10.1111/j.1365-263X.2011.01131.x.

21. Sjögren $U$, Figdor $D$, et al. Influence of infection at the time of root filling on the outcome of endodontic treatment of teeth with apical periodontitis. Int Endod J 1997;30(5):297-306. DOI: 10.1111/j.13652591.1997.tb00714.x.

22. Trope M, Delano EO, et al. Endodontic treatment of teeth with apical periodontitis: single vs. multivisit treatment. J Endod 1999;25(5): 345-350. DOI: 10.1016/S0099-2399(06)81169-6.

23. Weiger R, Rosendahl R, et al. Influence of calcium hydroxide intracanal dressings on the prognosis of teeth with endodontically induced periapical lesions. Int Endod J 2000;33(3):219-226. DOI: 10.1046/j.1365-2591.1999.00298.x.

24. Albashaireh Z, Alnegrish A. Postobturation pain after single-and multiple-visit endodontic therapy. A prospective study. J Dent 1998;26(3):227-232. DOI: 10.1016/S0300-5712(97)00006-7.

25. DiRenzo A, Gresla T, et al. Postoperative pain after 1-and 2-visit root canal therapy. Oral Surg Oral Med Oral Pathol Oral Radiol Endod 2002;93(5):605-610. DOI: 10.1067/moe.2002.121900.

26. Ghoddusi J, Javidi $M$, et al. Flare-ups incidence and severity after using calcium hydroxide as intracanal dressing. N Y State Dent J 2006;72(4):24-28.

27. Gesi A, Hakeberg M, et al. Incidence of periapical lesions and clinical symptoms after pulpectomy-a clinical and radiographic evaluation of 1- vs 2-session treatment. Oral Surg Oral Med Oral Pathol Oral Radiol Endod 2006;101(3):379-388. DOI: 10.1016/j.tripleo.2005.05.073.

28. Al-Negrish AR, Habahbeh R. Flare up rate related to root canal treatment of asymptomatic pulpally necrotic central incisor teeth in patients attending a military hospital. J Dent 2006;34(9):635-640. DOI: 10.1016/j.jdent.2005.12.007.

29. Peters OA, Barbakow F, et al. An analysis of endodontic treatment with three nickel- titanium rotary root canal preparation techniques. Int Endod J 2004;37(12):849-859. DOI: 10.1111/j.1365-2591.2004. 00882.x.

30. Mortazavi M, Mesbahi M. Comparison of zinc oxide and eugenol, and Vitapex for root canal treatment of necrotic primary teeth. Int J Paediatr Dent 2004;14(6):417-424. DOI: 10.1111/j.1365-263X.2004. 00544.x.

31. Nadkarni U, Damle SG. Comparative evaluation of calcium hydroxide and zinc oxide eugenol as root canal filling materials for primary molars: a clinical and radiographic study. J Indian Soc Pedod Prev Dent 2000;18(1):1-10.
32. Pinky C, Shashibhushan KK, et al. Endodontic treatment of necrosed primary teeth using two different combinations of antibacterial drugs: an in vivo study. J Indian Soc Pedod Prev Dent 2011;29(2): 121-127. DOI: 10.4103/0970-4388.84684.

33. Rabinowitch BZ. Pulp management in primary teeth. Oral Surg Oral Med Oral Pathol 1953;6(5):671-676. DOI: 10.1016/0030-4220(53) 90171-6.

34. Spedding RH. Root canal treatments for primary teeth. Dent Clin North Am 1973;17(1):105-124.

35. Cartwright HV, Bevans JL. Management of two abscessed primary molars in a four-year-old child: report of interesting case. ASDC $J$ Dent Child 1970;37(3):230-232.

36. Rifkin A. A simple, effective, safe technique for the root canal treatment of abscessed primary teeth. ASDC J Dent Child 1980;47(6):435-441.

37. Nakornchai $S$, Banditsing $P$, et al. Clinical evaluation of 3 Mix and vitapex as treatment options for pulpally involved primary molars. Int J Paediatr Dent 2010;20(3):214-221. DOI: 10.1111/j.1365263X.2010.01044.x.

38. Chen XX, Lin BC, et al. Degradation evaluation and success of pulpectomy with a modified primary root canal filling in primary molars. Beijing Da Xue Xue Bao 2015;47(3):529-535.

39. Subramaniam $P$, Gilhotra K. Endoflas, zinc oxide eugenol and metapex as root canal filling materials in primary molars-a comparative clinical study. J Clin Pediatr Dent 2011;35(4):365-369. DOI: 10.17796/ jcpd.35. 4.1377v06621143233.

40. Ramar K, Mungara J. Clinical and radiographic evaluation of pulpectomies using three root canal filling materials: an in vivo study. J Indian Soc Pedod Prev Dent 2010;28(1):25-29. DOI: 10.4103/09704388.60481.

41. Coll JA, Josell S, et al. Evaluation of a one-appointment formocresol pulpectomy technique for primary molars. Pediatr Dent 1985;7(2): 123-129.

42. O'Riordan MW, Coll J. Pulpectomy procedure for deciduous teeth with severe pulpal necrosis. J Am Dent Assoc 1979;99(3):480-482. DOI: 10.14219/jada.archive.1979.0310.

43. Molander A, Warfvinge J, et al. Clinical and radiographic evaluation of one- and two-visit endodontic treatment of asymptomatic necrotic teeth with apical periodontitis: a randomized clinical trial. J Endod 2007;33(10):1145-1148. DOI: 10.1016/j.joen.2007.07.005.

44. Penesis VA, Fitzgerald PI, et al. Outcome of one-visit and two-visit endodontic treatment of necrotic teeth with apical periodontitis: a randomized controlled trial with one-year evaluation. J Endod 2008;34(3):251-257. DOI: 10.1016/j.joen.2007.12.015.

45. Akbar I, Iqbal $A$, et al. Flare-up rate in molars with periapical radiolucency in one-visit vs two-visit endodontic treatment. J Contemp Dent Pract 2013;14(3):414-418. DOI: 10.5005/jp-journals10024-1337.

46. Singh S, Garg A. Incidence of post-operative pain after single visit and multiple visit root canal treatment: a randomized controlled trial. J Conserv Dent 2012;15(4):323-327. DOI: 10.4103/0972-0707. 101888.

47. Wong AW, Zhang S, et al. Incidence of post-obturation pain after single-visit vs multiple-visit non-surgical endodontic treatments. BMC Oral Health 2015;15:96. DOI: 10.1186/s12903-0150082-y. 